\title{
Use of the kojA promoter, involved in kojic acid biosynthesis, for polyketide production in Aspergillus oryzae: implications for long- term production
}

\author{
Koichi Tamano ${ }^{1,2^{*}}$, Mahoko Kuninaga ${ }^{3}$, Naoshi Kojima ${ }^{4}$, Myco Umemura ${ }^{2,3}$, Masayuki Machida ${ }^{1}$ and Hideaki Koike ${ }^{3}$
}

\begin{abstract}
Background: Aspergillus oryzae, a useful industrial filamentous fungus, produces limited varieties of secondary metabolites, such as kojic acid. Thus, for the production of valuable secondary metabolites by genetic engineering, the species is considered a clean host, enabling easy purification from cultured cells. A. oryzae has been evaluated for secondary metabolite production utilizing strong constitutive promoters of genes responsible for primary metabolism. However, secondary metabolites are typically produced by residual nutrition after microbial cells grow to the stationary phase and primary metabolism slows. We focused on a promoter of the secondary metabolism gene kojA, a component of the kojic acid biosynthetic gene cluster, for the production of other secondary metabolites by A. oryzae.
\end{abstract}

Results: A kojA disruptant that does not produce kojic acid was utilized as a host strain for production. Using this host strain, a mutant that expressed a polyketide synthase gene involved in polyketide secondary metabolite production under the kojA gene promoter was constructed. Then, polyketide production and polyketide synthase gene expression were observed every $24 \mathrm{~h}$ in liquid culture. From days 0 to 10 of culture, the polyketide was continuously produced, and the synthase gene expression was maintained. Therefore, the kojA promoter was activated, and it enabled the continuous production of polyketide for 10 days.

Conclusions: The combined use of the kojA gene promoter and a kojA disruptant proved useful for the continuous production of a polyketide secondary metabolite in A. oryzae. These findings suggest that this combination can be applied to other secondary metabolites for long-term production.

Keywords: Aspergillus oryzae, Kojic acid, Secondary metabolism, Transcriptional regulation, Polyketide

\section{Background}

Aspergillus oryzae is a useful industrial filamentous fungus; it has been utilized for the production of Japanese alcohol (sake), soy sauce (shoyu), soybean paste (miso), and other products since ancient periods in Japan. It has a strong ability to produce hydrolases, such as amylase,

\footnotetext{
* Correspondence: tamano-k@aist.go.jp

${ }^{1}$ Bioproduction Research Institute, National Institute of Advanced Industrial Science and Technology (AIST), 2-17-2-1 Tsukisamu-Higashi, Toyohira-ku, Sapporo, Hokkaido 062-8517, Japan

${ }^{2}$ AIST-Waseda University Computational Bio Big-Data Open Innovation Laboratory (CBBD-OIL), National Institute of Advanced Industrial Science and Technology (AIST), 5-20, Building 63, Nishi-waseda campus, Waseda University, 3-4-1 Okubo, Shinjuku-ku, Tokyo 169-8555, Japan Full list of author information is available at the end of the article
}

protease, and lipase. It is also beneficial with respect to safety owing to its lack of mycotoxin production and formally approved as a safe microorganism with the GRAS (Generally Recognized as Safe) status by FDA (Food and Drug Administration of USA). The whole genome sequence of the wild-type $A$. oryzae strain RIB40 was reported in 2005 [1], providing a basis for targeted gene disruption or overexpression $[2,3]$. In addition to enzymes, industrially valuable metabolite production has been attempted using $A$. oryzae by metabolic engineering via genetic modification technology [4-6].

A. oryzae produces a limited array of secondary metabolites, such as kojic acid $[7,8]$, and does not produce 
mycotoxins, such as aflatoxin and cyclopiazonic acid [9, 10]. In fact, heterologous production of secondary metabolites of polyketides, terpenes, and non-ribosomal peptides has ever been attempted in $A$. oryzae, and from the data presented here, it is indicated that none or only one peak of compounds with similar physical properties exist in the wild-type strain [11-14]. Thus, for the heterologous production of valuable secondary metabolites, we proposed $A$. oryzae to be adequate with respect to the ease of purification from cultured cells. In other words, it should be expected to be a clean host with limited endogenous secondary metabolites. Furthermore, knockout mutants of kojic acid biosynthesis genes are considered more effective hosts for secondary metabolite production than the wild-type strain because they do not produce any kojic acid.

Fungal secondary metabolites are required in the cells or spores for protection against ultraviolet (UV) and other microorganisms like bacteria, or for development [15]. Some of them possess medicinal properties and are actually used as pharmaceuticals such as penicillin, lovastatin, and cyclosporin $[15,16]$. Most secondary metabolites are categorized into three groups: polyketides, small peptides (e.g. non-ribosomal peptides), and terpenes [15, 17]. Polyketides and terpenes are synthesized from
acetyl-CoA and malonyl-CoA, and thus are characteristically composed of only carbon, oxygen, and hydrogen, but not nitrogen. Non-ribosomal peptides are synthesized from amino acids and thus contain nitrogen as well. Kojic acid shares the components of polyketides and terpenes; however, it is considered not to be categorized as either of them and considered to be synthesized through a different metabolic pathway [18].

In fact, for the first time, we previously identified genes responsible for kojic acid biosynthesis in A. oryzae [19]. They comprise kojA (AO090113000136), kojR (AO090113000137), and kojT (AO090113000138) and form a gene cluster on the chromosome (Fig. 1a) [19]. To date, it is revealed that the $k o j R$-translated product, KojR, acts as a transcription factor and can activate promoters of other cluster genes, namely $k o j A$ and $k o j T$ [20]. The kojA-translated product, KojA, is predicted to be an oxidoreductase and directly contributes to kojic acid biosynthesis using some sort of metabolite as a substrate. However, it does not have a motif sequence of polyketide synthase (PKS) and P450 that usually exist in polyketide and terpene biosynthesis gene clusters, respectively. The kojT-translated product, KojT, is predicted to function as a transporter located on the cell membrane and transport kojic acid to the extracellular

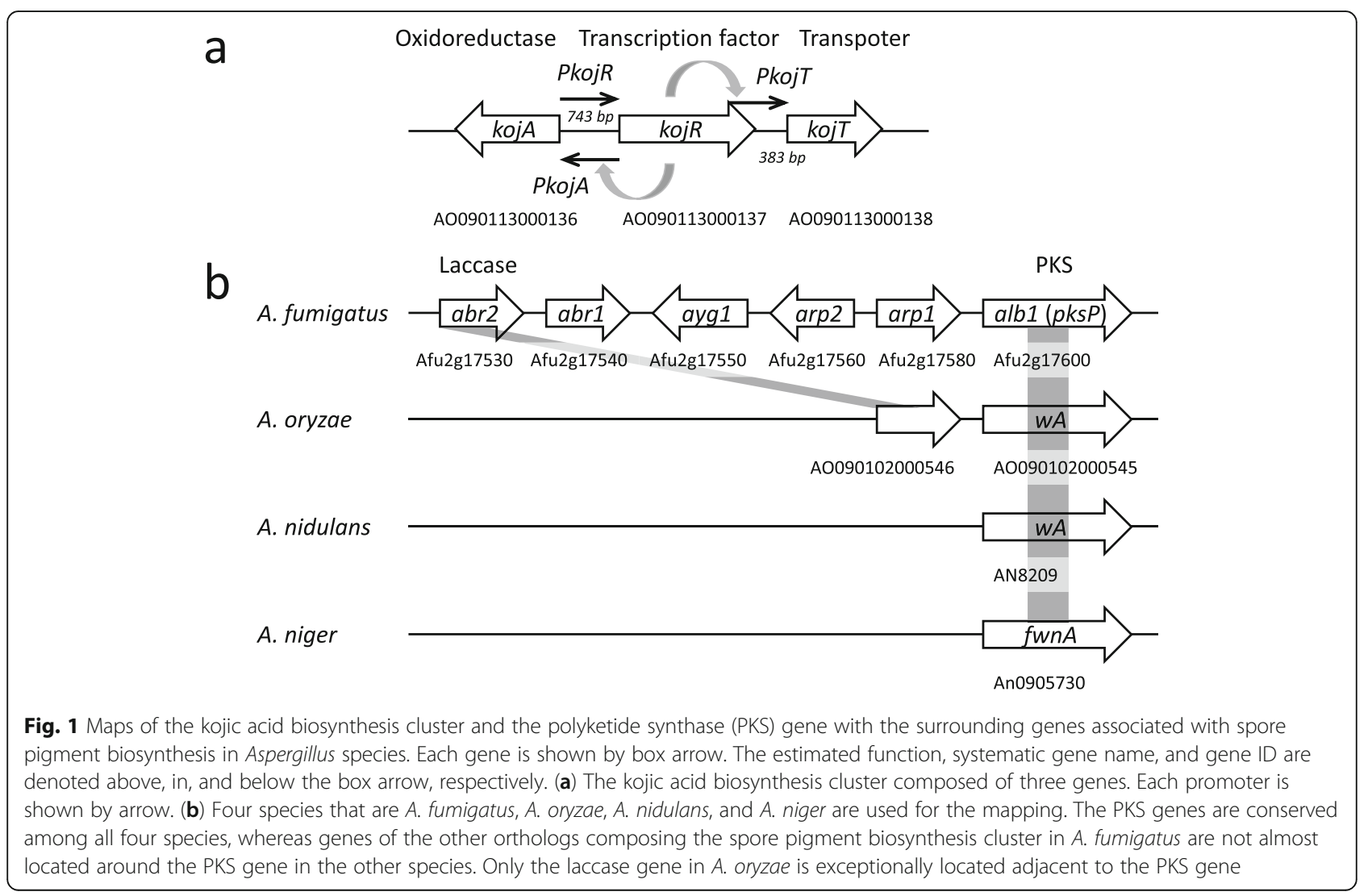


environment. These findings also indicate that kojic acid biosynthesis will be independent of polyketide and terpene biosyntheses.

We also analyzed the culture conditions under which this biosynthesis is activated. Kojic acid was continuously synthesized from culture day 3 to day 10 in kojic acid production liquid medium using residual glucose as a substrate after the nitrogen source was completely catabolized [19]. This implies that kojic acid biosynthesis is suppressed in the presence of a nitrogen source. In fact, the $k o j A$ gene expression was found to be repressed until the nitrogen source was used up [19]. Kojic acid is not essential for cell growth and thus is considered a secondary metabolite. Therefore, the promoters in the kojic acid biosynthesis gene cluster should exhibit lower activity levels than those of primary metabolism genes. However, because kojic acid is continuously produced, promoters of the genes involved in its biosynthesis are expected to be activated for a long period during production. In the stationary growth phase of $A$. oryzae, the primary metabolism diminishes, and kojic acid accumulates in the cells owing to its continuous production using the residual carbon source, glucose [19]. In such a situation, selective production of a metabolite seems to come into play, which will contribute to the easy and efficient recovery from cells. Therefore, in this study, we focused on the long-term production characteristics of kojic acid, and a promoter of $k o j A$ was evaluated for the long-term production of other secondary metabolites in $A$. oryzae. The polyketide synthase gene $w A$ of $A$. oryzae was chosen for the evaluation, because polyketide has the same composition as kojic acid and could be easily quantified by a specific ultraviolet-visible (UV-Vis) absorbance.

\section{Results}

Production of a polyketide YWA1 synthesized by $A$. oryzae wA under the kojA promoter in the kojA disruptant To begin with, we validated our assumption that the expression of primary metabolism genes attenuates toward the end of culture. The eno $A$ gene encoding the enolase enzyme in glycolysis is considered constitutive gene and has ever been used for heterologous gene expression [21]. It was selected for the validation. The expression was certainly decreased as the culture of RIB40 proceeded in the kojic acid production medium (Additional file 1: Figure S1). Thus, our assumption seemed adequate, and as mentioned above, we then chose a polyketide secondary metabolite for evaluating the applicability of the $k o j A$ promoter to the production. It is because the $k o j A$ promoter activation is dependent on a nitrogen-limited culture environment. Polyketide does not contain nitrogen in the molecule, and thus it was considered appropriate for evaluating the expression system by the kojA promoter. In the $A$. oryzae genome, orthologues of genes involved in the biosynthesis of the spore pigment were predicted (Fig. 1b). A PKS encoded by $w A$ (AO090102000545), an ortholog of alb1 (Afu2g17600) in Aspergillus fumigatus Af293, fwnA (An09g05730) in Aspergillus niger CBS 513.88, and wA (AN8209) in Aspergillus nidulans A4, is likely involved in the initial step of biosynthesis of spore pigments using malonyl-CoAs as substrates. According to a previous study based on $A$. nidulans (Fig. 2), the product of $A$. nidulans $w A$ is a spore pigment precursor; however, it has a yellow color and is named YWA1 [22]. The product of $A$. oryzae $w A$ was expected to be similar to that of $A$. nidulans $w A$ and expected to be measured by absorbance easily in the experimental technique. Thus, by replacing the promoter of $w A$ with that of $k o j A$, production of the polyketide derived from $A$. oryzae $w A$ under the regulation of the kojA promoter was attempted in $A$. oryzae.

A $k o j A$ disruptant constructed in our previous study and named $\Delta k o j A \_n i a D$ - [19] was used as a parental host strain for constructing the mutant strain expressing $w A$ under the control of the $k o j A$ promoter. The $\Delta k o j A_{-}$ niaD- strain does not produce kojic acid and should produce only small amounts of other secondary metabolites. The $\Delta k o j A \_n i a D$ - strain is also an auxotroph of nitrate due to a mutation in a nitrate reductase gene (niaD, AO090012001035); thus, niaD was used as a selectable marker. A DNA cassette for the expression of A. oryzae $w A$ under the $k o j A$ promoter (Additional file 1: Figure S2) was prepared by fusion PCR as described in the Methods section. It was introduced to the $\triangle k o j A \_n i a D$ strain by transformation, and the transformant colonies were subjected to single spore isolation and verified by both PCR and Southern hybridization. Homologous recombination was confirmed at only the targeted $w A$ locus by Southern hybridization (Fig. 3a-b). The mutant with promoter replacement was named $\Delta k o j A \_P k o j A:$ : $w A$. Similarly, the entire intact niaD gene was prepared by PCR using RIB40 chromosomal DNA as a template (Additional file 1: Figure S3), and was introduced to the $\triangle k o j A \_n i a D$ - strain to construct a control mutant strain. The niaD-complemented kojA disruptant was confirmed by Southern hybridization (Fig. 3c-d) and was named $\triangle k o j A$. Both mutant strains were cultured on kojic acid production agar. The $\triangle k o j A$ strain showed yellow-green spores, similar to the spores of the wild-type strain, whereas the $\triangle k o j A \_P k o j A:: w A$ strain showed white spores (Fig. 4a, upper row). In contrast, the agar became yellowish only for the $\triangle k o j A \_P k o j A:: w A$ strain (Fig. 4A, lower row). When cultured in kojic acid production liquid medium, the culture supernatant of the $\Delta k o j A_{-}$ $P k o j A:: w A$ strain became more yellowish than the supernatant of the $\triangle k o j A$ strain (Fig. 4b). Considering that this 


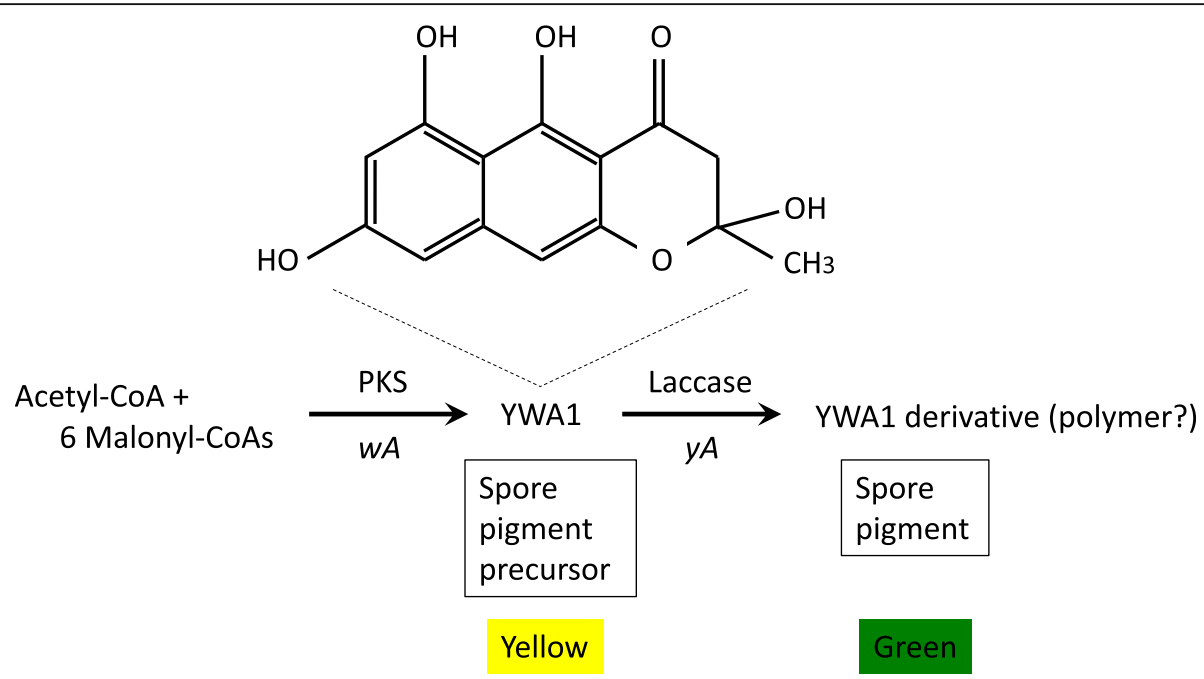

Fig. 2 Illustration of biosynthetic pathway of spore pigment in A. nidulans. The first step is performed by PKS encoded by A. nidulans wA. The polyketide named YWA1 is synthesized and the structure is drawn above. The second step is performed by a laccase encoded by $y$ A. The similar pathway seems to be there in $A$. oryzae

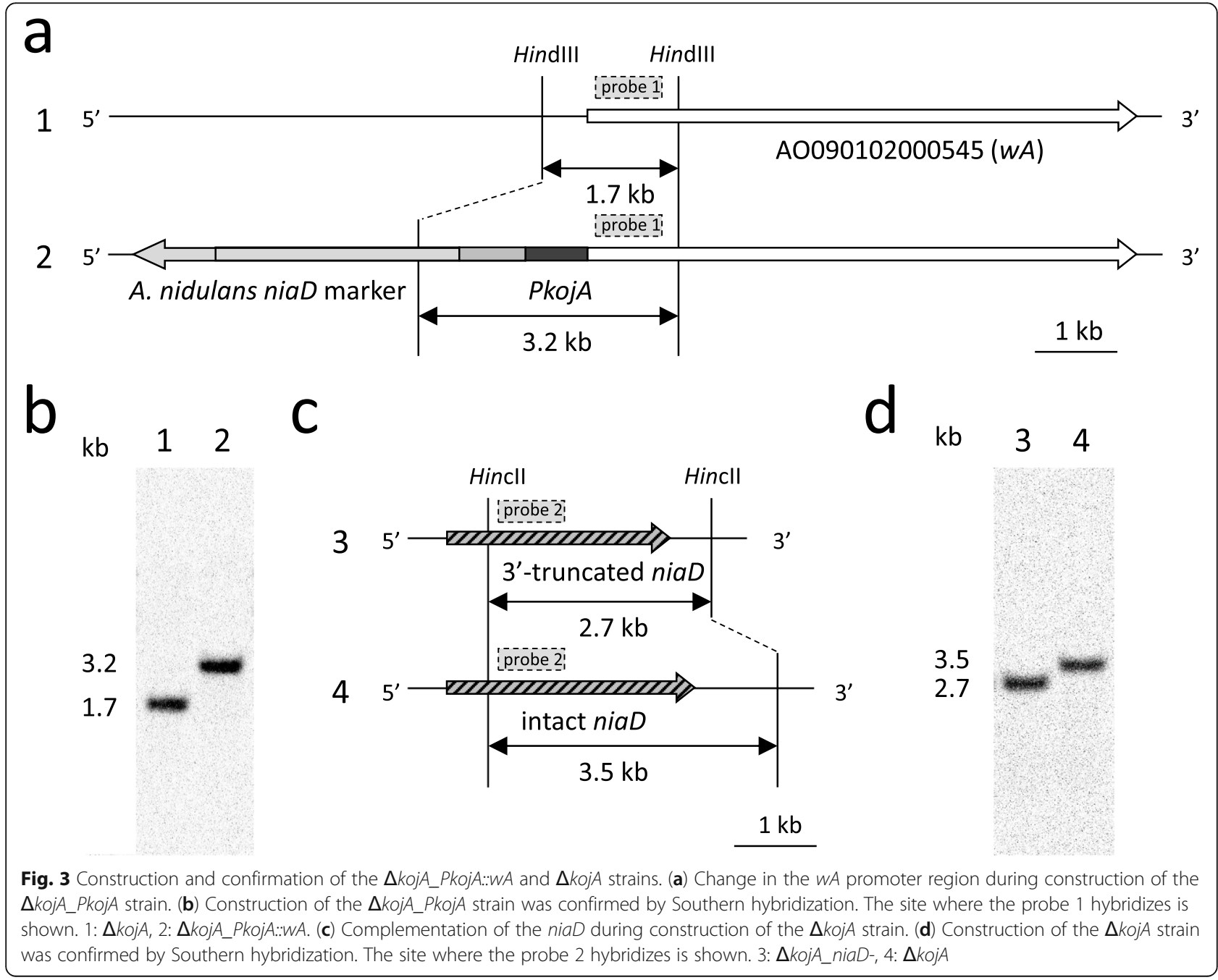




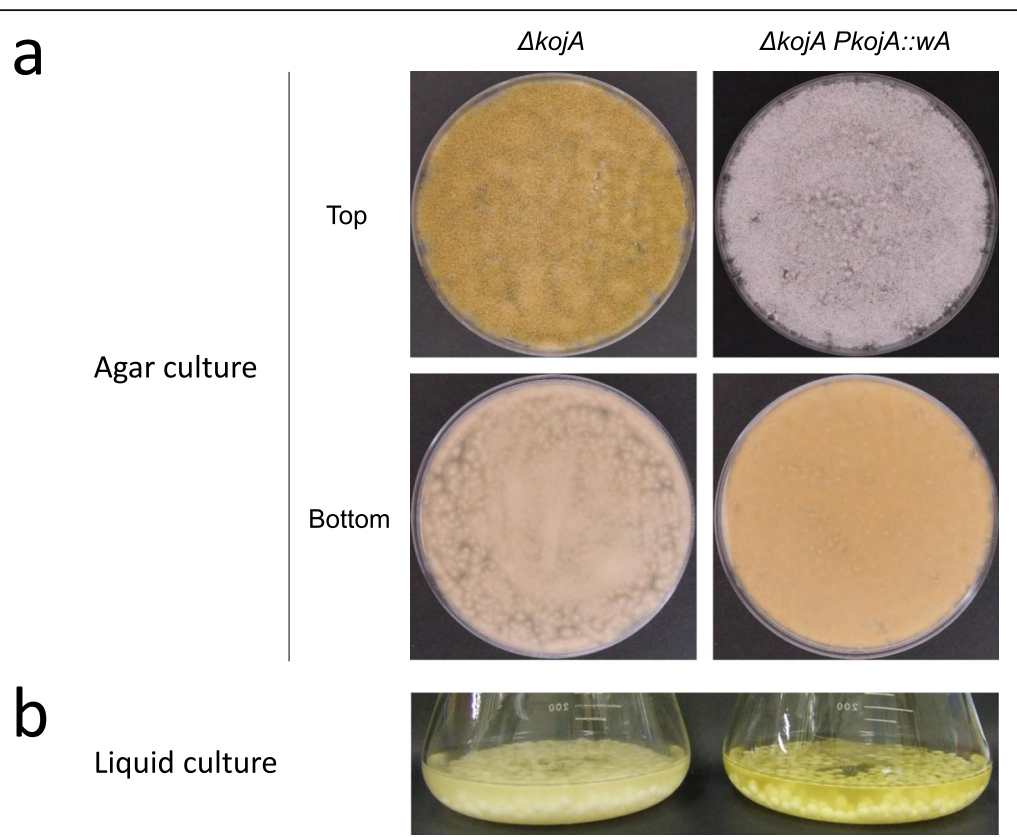

Fig. 4 Images of the $\triangle k o j A$ (left) and the $\triangle k o j A \_P k o j A:: w A$ (right) strains. (a) Image obtained after incubation at $30^{\circ} \mathrm{C}$ for 7 days of the $C D$ agar plates where $5 \times 10^{5}$ spores were spread-inoculated. Images of plates taken from the top (upper row) or the bottom (lower row) are shown. (b) Image of the liquid culture after cultivation at $30^{\circ} \mathrm{C}$ at $200 \mathrm{rpm}$ for 5 days

yellow color was derived from PKS, extraction and confirmation of the yellow-colored compound were attempted using ethyl acetate. The UV-Vis spectrum corresponded to that of the YWA1 standard (Additional file 1: Figures S4). A UPLC-MS analysis was also performed for the sample and the standard. The retention time of the major peak at 4.0 min observed at 280 $\mathrm{nm}$ by UPLC superimposed with that of YWA1 standard (Fig. 5a). In addition, the MS chromatogram of sample compounds whose $\mathrm{m} / \mathrm{z}$ was 277 was identical to that of the YWA1 standard (Fig. 5b). Hence, taken together, the yellow-colored compound produced by the $\Delta k o j A_{-}$ $P k o j A:: w A$ strain was attributed to the polyketide synthase encoded by $w A$ and concluded to be YWA1.

\section{Time-course shifts in $w A$ expression under the kojA promoter and the polyketide yield}

The activation of the $k o j A$ promoter was evaluated in the $\triangle k o j A \_P k o j A:: w A$ strain. The expression level of $w A$ and the YWA1 yield were measured every $24 \mathrm{~h}$ in a stepwise manner from the beginning of the culture period until day 10. As determined by qRT-PCR, $w A$ remained active from day 2 to day 10 of the culture period (Fig. 6). The expression level exhibited a peak on day 5 and decreased after day 9, but the differences were minimal. Therefore, the $k o j A$ promoter was active up to day 10 of the culture period. In the control $\Delta k o j A$ strain, the expression level of $w A$ showed a similar trend. As a result, $w A$ expression of the $\triangle k o j A$ strain was approximately
0.05 fold or less of that of $\triangle k o j A_{-} P k o j A:: w A$ strain in each culture period. This suggests that $w A$ was overexpressed by the kojA promoter in the $\triangle k_{0 j} A_{-} P k o j A:: w A$ strain.

Next, YWA1 was measured by estimates of absorbance, and then the YWA1 amount produced by unit gram of dried cells was evaluated. An absorbance peak at $406 \mathrm{~nm}$ was detected by UV-Vis spectrometry (Additional file 1: Figure S4); accordingly, absorbance at $406 \mathrm{~nm}$ in the culture supernatant was measured every $24 \mathrm{~h}$ from the beginning of the culture period until day 10. Absorbance of the culture medium before inoculation was then subtracted from that on each culture period to remove background, and the subtracted absorbance was subsequently divided by the weight of dried cells to evaluate the amount of YWA1 produced by the same number of cells. It was observed that YWA1 was continuously produced from day 1 to day 10; production was maintained during longterm liquid culture (Fig. 7). The YWA1 amount per gram of dried cells in the $\triangle k o j A \_P k o j A:: w A$ strain was 10.6-fold more than that in the control strain. The results corresponded to the enhanced $w A$ expression level as shown in Fig. 6, indicating that the kojA promoter is useful for high and long-term production. As in the case of eno $A$ (Additional file 1: Figure S1), expression of constitutive genes is generally considered to attenuate toward the stationary growth phase. Though we do not directly compare the case of $w A$ expression under between $k o j A$ promoter and enoA promoter, because high and long-term 


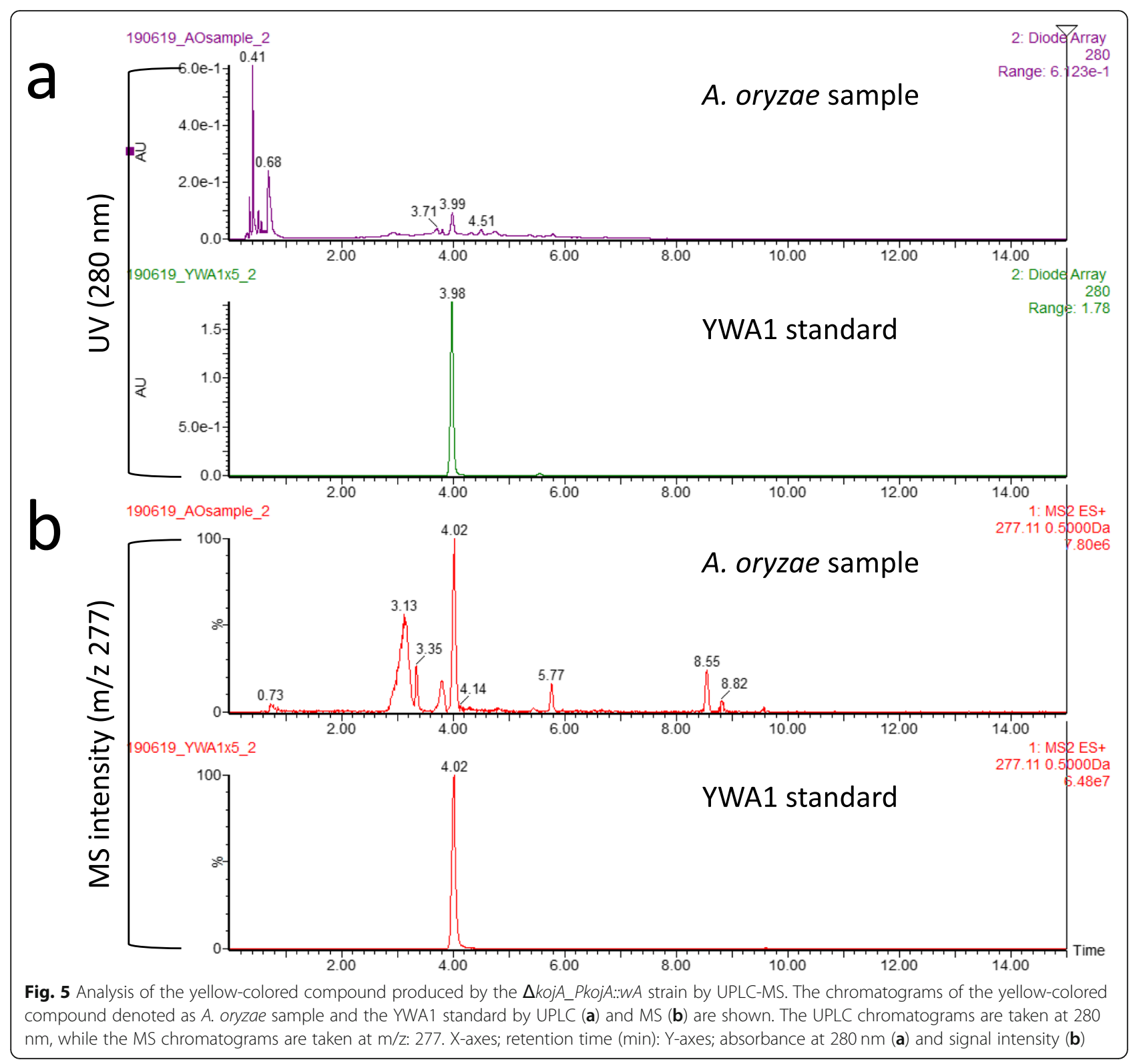

production was observed in case of YWA1 in this study, the $k o j A$ promoter seems to be useful for such a purpose.

\section{Discussion}

In this study, we showed that the $k o j A$ promoter enabled the continuous production of polyketide YWA1 encoded by $w A$ for 10 days in $A$. oryzae. As shown in Fig. 1b, in spite of conservation of the $w A$ orthologs between four Aspergillus species, the other orthologs included in the melanin synthesis cluster of $A$. fumigatus do not exist or are located far apart from the $w A$ ortholog on the genome in the other three Aspergillus species. Only the laccase gene ortholog (AO090102000546) exists adjacent to $w A$ on the $A$. oryzae genome. However, another gene involved in the spore pigment biosynthesis named $y A$
(AN6635) is present on the A. nidulans genome, which is located far away from $A$. nidulans $w A$ on the genome. The $y A$ encodes laccase as well and is reported to generate green-colored spore pigments, probably via polymerization of YWA1 through the laccase function (Fig. 2). However, the $y A$ homolog of A. oryzae is AO090011000755 and is different from the ortholog. Thus, there seem to be two laccase candidate genes involved in the spore pigment biosynthesis in A. oryzae. A fact which predominantly functions in it should be researched hereafter.

Because of the white spores of the $\triangle k o j A \_P k o j A:: w A$ strain, the $k o j A$ promoter may be active only in hyphae or even in spores at lower level than the RIB40. Because the production of valuable enzymes and compounds by $A$. oryzae is performed in liquid culture, the phenomenon of 


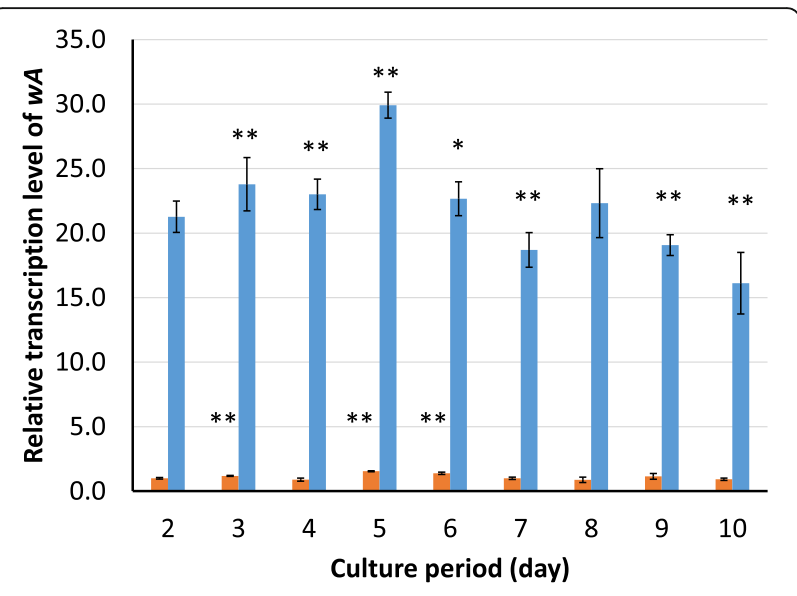

Fig. 6 Time-course shift in A. oryzae $W A$ expression. Expression levels of $W A$ in the wild-type RIB40 (orange) and the $\triangle$ kojA_PkojA::WA (blue) strains at each time point relative to that on day 2 of RIB40 are shown. Error bars represent mean \pm standard deviation $(n=3)$.

The result of a significance test (Student's t-test) between day 2 and the other date is shown above each bar. *and ** represent $p<0.05$ and $p<0.01$, respectively

whitish spores will not be a drawback when using the kojA promoter for production.

Because YWA1 does not contain nitrogen, the production system using the $k o j A$ promoter in the $k o j A$ disruptant could be applied to the production of other compounds lacking nitrogen, like polyketides. On the other hand, because this production system is undertaken in nitrogen

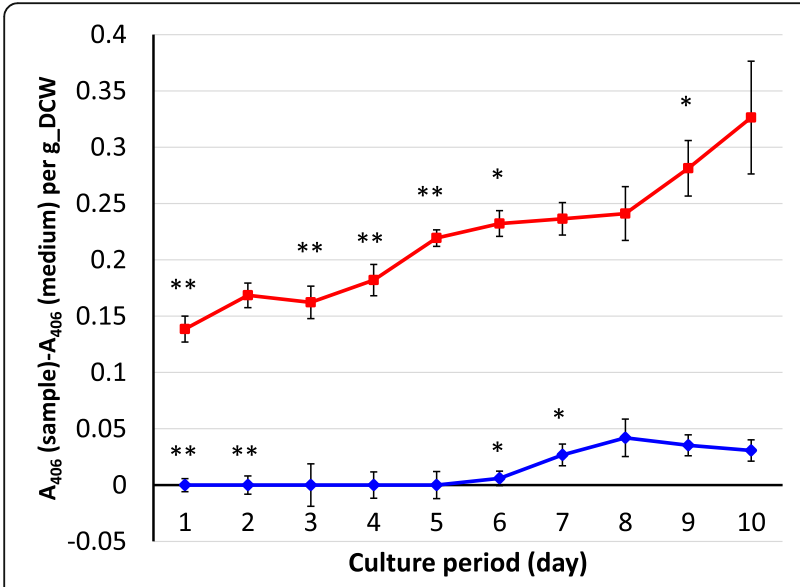

Fig. 7 Time-course shift in the A. oryzae WA product YWA1 secreted by the $\triangle$ kojA_PkojA::WA strain. The product yield was measured by specific absorbance at $406 \mathrm{~nm}$ of the culture supernatant. Absorbance of the culture supernatant at each culture period was subtracted by that of culture medium without inoculation, followed by division by dry cell weight of hyphae. The calculated value was monitored for both the $\triangle$ kojA_PkojA.:WA strain (red) and the control $\triangle$ kojA strain (blue). Error bars represent mean \pm standard deviation $(n=3)$. The result of a significance test (Student's t-test) between the day 0 and the other date is shown above each bar. ${ }^{*}$ and ${ }^{* *}$ represent $p<0.05$ and $p<0.01$, respectively source-deficient condition, it was considered difficult to be applied to the production of compounds containing nitrogen, such as non-ribosomal peptides. However, unexpectedly, YWA1 production by the $\triangle k o j A \_P k o j A:: w A$ strain was observed from day 1 of the culture period (Fig. 7). We expected production to begin on day 3 , after the nitrogen source was catabolized. In fact, $w A$ expression level under the $k o j A$ promoter at day 2 of the culture period was already high in the $\triangle k o j A \_P k o j A:: w A$ strain, while $k o j A$ expression level under the $k o j A$ promoter at the day 2 was quite low in the RIB40 strain (Additional file 1: Figure S5). The data would imply that the expression in the $\Delta k o j A_{-}$ $P k o j A:: w A$ strain was actually independent of nitrogen starvation. The reason for this unexpected result is unclear and should be evaluated in future studies. There might be additional regulatory mechanisms apart from that utilizing the nitrogen source. For example, the $k o j A$ promoter was attached to the $w A$ locus, where epigenetic modifications might be different from those at the original $k o j A$ locus. However, with respect to metabolite production, expression from the early stage of culture is advantageous and is not an issue. In addition, since the $k o j A$ promoter seemed active even at culture period day 1 when nitrogen source remained, although it was not exemplified in this study, the expression system with the $k o j A$ promoter could be applied to non-ribosomal peptide production where plenty of nitrogen source will be required in culture.

Polyketide final products are generally synthesized not only by PKS but also by several modifying enzymes. Thus, for increase in production, several genes of enzymes including PKS involved in the synthesis will need to be overexpressed. In such an attempt, multiple uses of the $k o j A$ promoter for expressing these genes will be one possible solution. If the $k o j A$ promoter was used redundantly, its transcriptional factor, KojR, would get shortage in the cell. In such a case, it will be effective to use the $k o j R$ gene multiply as well. Fortunately, as shown in Fig. 1a, the kojA promoter shares the locus with the $k o j R$ promoter but in the opposite direction. In other words, it is a bidirectional promoter. Thus, only extending the DNA region used for replacement with the $k o j A$ promoter in this study to the end of $k o j R$ coding sequence will enable not only the replacement but also additional $k o j R$ arrangement on the genome.

\section{Conclusions}

The combination of the $k o j A$ gene promoter and the $k o j A$ disruptant was useful for continuous production of a polyketide secondary metabolite until day 10 of the culture period. The results suggested that this combination is applicable to other secondary metabolites for long-term production in A. oryzae. 


\section{Methods}

\section{Fungal strains and culture}

A. oryzae wild-type strain RIB40 and its mutants were used. The RIB40 strain was distributed by the National Research Institute of Brewing (NRIB, Hiroshima, Japan) and approved of use for research purposes [1]. For genetic engineering, an $A$. oryzae $\Delta k o j A \_n i a D$ - strain with the genotype $\triangle$ ligD::ptrA $\triangle$ pyrG::AnsC $\triangle$ kojA::AnpyrG (niaD-), derived from NS4 [23, 24], the niaD- and $s C$ auxotrophic mutant of RIB40, and constructed in our previous study [19], was used. The $\Delta k o j A \_n i a D$ - strain was originally removed capability of non-homologous end joining (NHEJ) due to knockout of a ligase gene ligD responsible for NHEJ [23]. Thus, only homologous recombination can be performed in the strain and the efficiency is quite high.

Each $A$. oryzae strain constructed by genetic engineering was maintained at $30^{\circ} \mathrm{C}$ on Czapek-Dox (CD) agar medium [25], and the spores were stored in $20 \%(\mathrm{w} / \mathrm{v})$ glycerol at $-80{ }^{\circ} \mathrm{C}$ until use. To test YWA1 production under the $k o j A$ promoter, the spores were inoculated on kojic acid production agar medium $(0.25 \%$ yeast extract, $0.1 \% \mathrm{~K}_{2} \mathrm{HPO}_{4}, 0.05 \% \mathrm{MgSO}_{4}-7 \mathrm{H}_{2} \mathrm{O}, 10 \%$ glucose, and $1.5 \%$ agar; $\mathrm{pH}$ adjusted to 6.0 before autoclaving) [19] and harvested. Then, $2.5 \times 10^{7}$ spores were inoculated into $50 \mathrm{~mL}$ of the kojic acid production liquid medium and cultured at $30{ }^{\circ} \mathrm{C}$ and $200 \mathrm{rpm}$ for up to 10 days.

\section{Construction of the $A$. oryzae mutant strains}

All primers used in this study are listed in Additional file 1: Table S1. The promoter-replaced strain, $\Delta k o j A$ $P k o j A:: w A$, was constructed as follows. The $\Delta k o j A \_n i a D-$ strain, a kojA disruptant lacking niaD constructed in our previous study [19], was transformed with the DNA fragment for $w A$ expression under the kojA promoter. The DNA fragment was constructed by PCR and the subsequent fusion PCR using the KOD-PLUS DNA polymerase (Toyobo, Osaka, Japan) [26]. Two 1-kb DNA fragments of the promoter and the coding sequence from the start codon of $w A$ were amplified with LU/LL and RU/RL primer pairs from the RIB40 genomic DNA template. Similarly, a $1-\mathrm{kb}$ DNA fragment of the kojA promoter was amplified with PU_PkojA/PL_PkojA primer pair from the RIB40 genomic DNA. A $4.7-\mathrm{kb}$ DNA fragment of AnniaD including the promoter and the terminator was amplified as a selectable marker with PU_AnniaD/PL_AnniaD primer pair from A. nidulans A851 genomic DNA. These four DNA fragments were mixed and were subjected to fusion PCR with LU/RL primer pair (Additional file 1: Figure S2). The resultant fragment harboring the $w A$ gene portion in which the promoter was replaced with the $k o j A$ promoter was introduced to the $\triangle k o j A \_n i a D$ - strain by transformation. The $\Delta k o j A \_P k o j A:: w A$ stain was selected by picking transformants grown on CD agar medium and confirmed by both PCR with LU/RL primer pair and Southern hybridization.

The control strain, $\Delta k o j A$, was made by complementing the niaD gene obtained from $A$. oryzae with the $\Delta k o j A$ niaD- strain. A 5.4-kb DNA fragment of niaD including 0.9-1 kb regions of the promoter and the terminator was amplified with PU_niaD/PL_niaD primer pair from $A$. oryzae RIB40 genomic DNA and used for the transformation of $\Delta k o j A \_n i a D$ - (Additional file 1: Figure S3). The $\Delta k o j A$ strain was selected by picking transformants grown on $C D$ agar medium and confirmed by PCR with PU_niaD/PL_ niaD primer pair.

\section{Southern hybridization}

Aliquots $(3 \mu \mathrm{g})$ of genomic DNA were digested with the restriction enzyme (HindIII or HincII), fractionated on a $0.7 \%$ agarose gel. Digoxigenin (DIG)-labeled probe (approximately $800 \mathrm{bp}$ ) was prepared using a PCR DIG Probe Synthesis Kit (Roche Applied Science, Mannheim, Germany) with the primers SBU and SBL. The probe preparation, hybridization, and signal detection were performed as described previously [3].

\section{Extraction of the yellow-colored compound, confirmation by UV-Vis spectrometry and UPLC-MS, and the quantification}

To analyze the yellow-colored compound secreted from the $\Delta k o j A \_P k o j A:: w A$ strain, $30 \mathrm{~mL}$ of culture supernatant after liquid culture was extracted with $20 \mathrm{~mL}$ of ethyl acetate. The yellow-colored compound was extracted in the ethyl acetate fraction. The separated ethyl acetate fraction was concentrated in vacuo using a rotary evaporator, and the residue was dissolved in ethanol $(1.5 \mathrm{~mL})$. A small portion was added to $1 \mathrm{~mL}$ of Milli-Q water, and then UV-Vis spectrometry was performed by monitoring absorbance in the wavelength range from $200 \mathrm{~nm}$ to $500 \mathrm{~nm}$ using a UV-Vis spectrophotometer (Shimadzu UV-1800; Tokyo, Japan). Then, another small portion was redissolved with ethyl acetate and $1 \mu \mathrm{L}$ of the solution was evaluated by ultraperformance liquid chromatography (UPLC)-mass spectrometry (MS) using the Waters Xevo TQD, Acquity UPLC $\mathrm{H}$ class equipped with an Waters Acquity UPLC BEH C18 $(1.7 \mu \mathrm{m}, 2.1 \times 50 \mathrm{~mm})$ column (Waters, Milford, MA, USA). 15\% acetonitrile in Milli-Q water over 0-0.5 min and a subsequent linear gradient of 15 to $100 \%$ acetonitrile in Milli-Q water containing $0.1 \%$ formic acid over $0.5-15 \mathrm{~min}$ at a flow rate of $0.4 \mathrm{~mL} / \mathrm{min}$ were used as the mobile phase. The column temperature was set to $40{ }^{\circ} \mathrm{C}$. The yellow-colored compound was detected by monitoring absorbance at $280 \mathrm{~nm}$. Because other compounds with absorbance at $280 \mathrm{~nm}$, such as nucleic acids and proteins, were removed at the extraction step 
with ethyl acetate, the yellow-colored compound was detected with high sensitivity.

For the quantification of the yellow-colored compound, $500 \mu \mathrm{L}$ of the culture supernatant was sampled and filtered using Ultrafree-MC-HV Centrifugal Filters DuraporePVDF $0.45 \mu \mathrm{m}$ (Millipore, Burlington, MA, USA). Then, absorbance at $406 \mathrm{~nm}$ was measured according to the second largest peak of UV spectrum at this wavelength. Since the culture supernatant was directly measured, this wavelength was used for precise quantification.

As a standard, the naphthopyrone compound YWA1, a product of polyketide synthase encoded by $w A$ of $A$. nidulans, was similarly evaluated by both UV-Vis spectrometry and UPLC-MS [22].

\section{Quantitative reverse transcription polymerase chain reaction ( $q R T-P C R)$}

Primers named "real_fwd" and "real_rev" were used for the quantification of gene expression levels. Expression level of the histone gene (AO090012000496) was used for normalizing the expression level of each gene [27]. RNA extraction and the subsequent qRT-PCR were performed as described previously [3].

\section{Supplementary information}

Supplementary information accompanies this paper at https://doi.org/10. 1186/s12896-019-0567-x.

\begin{abstract}
Additional file 1: Figure S1. Time-course shift in enoA expression in the wild-type RIB40 strain. Expression level at each time point relative to that on day 2 of RIB40 is shown. Error bars represent mean \pm standard deviation $(n=3)$. The result of a significance test (Student's t-test) between day 2 and the other date is shown above each bar. ${ }^{* *}$ represent $p<0.01$. Figure S2. Construction of the DNA fragment for replacement of WA promoter with kojA promoter in A. oryzae. The 7794 bp long DNA fragment was constructed for the replacement. Primers used for the construction and clone check are shown as arrows. Figure S3. Construction of the DNA fragment for complementing the niaD gene to the A. oryzae $\Delta$ ko$j A \_n i a D$ - where $769 \mathrm{bp}$ long region was deleted at the original niaD locus. The 5398 bp long DNA fragment was constructed. Primers used for the construction and clone check are shown as arrows. Figure S4. UV-Vis spectra of the YWA1 standard (A) and yellow-colored compound produced by the $\triangle k 0 j A \_P k o j A .: w A$ strain (B). Fig. S5. Time-course shift of the kojA promoter activities. The kojA promoter activity evaluated as kojA expression level at the kojA locus in the wild-type RIB40 strain (orange) and the one evaluated as WA expression level at the WA locus in the $\triangle$ kojA_PkojA::WA strain (blue) are compared. Both expression levels are normalized by the expression level of the histone gene as an internal standard and then compensated expression level at each time point relative to that on day 2 of RIB40 is shown. Error bars represent standard deviation $(n=3)$. Result of a significance test (Student's t-test) between day 2 of RIB40 and the other date is shown above each bar. ** represent $p<0.01$. Table $\mathbf{S 1}$. DNA primers used in this study. Tails of primers are shown in lower case letters.
\end{abstract}

\section{Abbreviations}

A. nidulans: Aspergillus nidulans; A. niger: Aspergillus niger; A. oryzae: Aspergillus oryzae; CD: Czapek-Dox; cDNA: complementary DNA; DIG: Digoxigenin; HPLC: High performance liquid chromatography; PCR: Polymerase chain reaction; PKS: Polyketide synthase; qRT-PCR: quantitative reverse transcription polymerase chain reaction; UPLC-MS: Ultraperformance liquid chromatography-mass spectrometry; UV-Vis: Ultraviolet-visible

\section{Acknowledgements}

The authors would like to thank Dr. Isao Fujii for kindly providing the YWA1 standard. The authors are also grateful to Dr. Tomomi Toda for experimental assistance.

\section{Authors' contributions}

$K T, M U, M M$, and HK conceived the experiments. KT, MK, NK, and MU performed experiments and analyzed the data. KT, NK, MU, and HK wrote the manuscript. All authors read and approved of the final manuscript.

\section{Funding}

This work was supported by a Grant-in-Aid for Scientific Research on Innovative Areas (JSPS KAKENHI Grant Number 22108007) and a Grant-in-Aid for Scientific Research (JSPS KAKENHI Grant Number 16 K01930), from the Ministry of Education, Culture, Sports, Science and Technology (MEXT) of Japan. The funding body had no role in the design of the study, the collection, analysis, and interpretations of data and in writing the manuscript.

\section{Availability of data and materials}

The datasets used and/or analyzed during the current study are available from the corresponding author on reasonable request.

Ethics approval and consent to participate

Not applicable.

\section{Consent for publication}

Not applicable.

\section{Competing interests}

The authors declare that they have no competing interests.

\section{Author details}

${ }^{1}$ Bioproduction Research Institute, National Institute of Advanced Industrial Science and Technology (AIST), 2-17-2-1 Tsukisamu-Higashi, Toyohira-ku, Sapporo, Hokkaido 062-8517, Japan. ${ }^{2}$ AIST-Waseda University Computational Bio Big-Data Open Innovation Laboratory (CBBD-OIL), National Institute of Advanced Industrial Science and Technology (AIST), 5-20, Building 63, Nishi-waseda campus, Waseda University, 3-4-1 Okubo, Shinjuku-ku, Tokyo 169-8555, Japan. ${ }^{3}$ Bioproduction Research Institute, National Institute of Advanced Industrial Science and Technology (AIST), Central 6, 1-1-1 Higashi, Tsukuba, Ibaraki 305-8566, Japan. ${ }^{4}$ Biomedical Research Institute, National Institute of Advanced Industrial Science and Technology (AIST), Central 6 , 1-1-1 Higashi, Tsukuba, Ibaraki 305-8566, Japan.

Received: 4 February 2019 Accepted: 10 October 2019

Published online: 26 October 2019

\section{References}

1. Machida M, Asai K, Sano M, Tanaka T, Kumagai T, Terai G, Kusumoto K, Arima T, et al. Genome sequencing and analysis of Aspergillus oryzae. Nature. 2005:438:1157-61.

2. Maruyama J, Kitamoto K. Targeted gene disruption in Koji mold Aspergillus oryzae. Methods Mol Biol. 2011;765:447-56.

3. Tamano K, Miura A. Further increased production of free fatty acids by overexpressing a predicted transketolase gene of the pentose phosphate pathway in Aspergillus oryzae faaA disruptant. Biosci Biotechnol Biochem. 2016;80:1829-35.

4. Brown SH, Bashkirova L, Berka R, Chandler T, Doty T, McCall K, McCulloch M, McFarland S, et al. Metabolic engineering of Aspergillus oryzae NRRL 3488 for increased production of L-malic acid. Appl Microbiol Biotechnol. 2013;97:8903-12.

5. Ichinose S, Tanaka M, Shintani T, Gomi K. Improved a-amylase production by Aspergillus oryzae after a double deletion of genes involved in carbon catabolite repression. Appl Microbiol Biotechnol. 2014;98:335-43.

6. Tamano K, Cox RS 3rd, Tsuge K, Miura A, Itoh A, Ishii J, Tamura T, Kondo A, et al. Heterologous production of free dihomo- - -linolenic acid by Aspergillus oryzae and its extracellular release via surfactant supplementation. J Biosci Bioeng. in press.

7. Frisvad JC, Møller LLH, Larsen TO, Kumar R, Arnau J. Safety of the fungal workhorses of industrial biotechnology: update on the mycotoxin and 
secondary metabolite potential of Aspergillus niger, Aspergillus oryzae, and Trichoderma reesei. Appl Microbiol Biotechnol. 2018;102:9481-515.

8. Sano M. Aspergillus oryzae nrtA affects kojic acid production. Biosci Biotechnol Biochem. 2016:80:1776-80.

9. Tominaga M, Lee YH, Hayashi R, Suzuki Y, Yamada O, Sakamoto K, Gotoh K, Akita O. Molecular analysis of an inactive aflatoxin biosynthesis gene cluster in Aspergillus oryzae RIB strains. Appl Environ Microbiol. 2006;72:484-90.

10. Tokuoka M, Seshime Y, Fujii I, Kitamoto K, Takahashi T, Koyama Y. Identification of a novel polyketide synthase-nonribosomal peptide synthetase (PKS-NRPS) gene required for the biosynthesis of cyclopiazonic acid in Aspergillus oryzae. Fungal Genet Biol. 2008:45:1608-15.

11. Kan E, Katsuyama Y, Maruyama Jl, Tamano K, Koyama Y, Ohnishi Y. Production of the plant polyketide curcumin in Aspergillus oryzae: strengthening malonyl-CoA supply for yield improvement. Biosci Biotechnol Biochem. 2019:83:1372-81.

12. Okada M, Matsuda Y, Mitsuhashi T, Hoshino S, Mori T, Nakagawa K, Quan Z, Qin B, et al. Genome-based discovery of an unprecedented cyclization mode in fungal Sesterterpenoid biosynthesis. J Am Chem Soc. 2016;138: 10011-8.

13. Tagami K, Liu C, Minami A, Noike M, Isaka T, Fueki S, Shichijo Y, Toshima H, et al. Reconstitution of biosynthetic machinery for indole-diterpene paxilline in Aspergillus oryzae. J Am Chem Soc. 2013;135:1260-3.

14. Yoshimi A, Yamaguchi S, Fujioka T, Kawai K, Gomi K, Machida M, Abe K. Heterologous production of a novel cyclic peptide compound, KK-1, in Aspergillus oryzae. Front Microbiol. 2018:9:690.

15. Keller NP. Fungal secondary metabolism: regulation, function and drug discovery. Nat Rev Microbiol. 2019;17:167-80.

16. Tamano K. Enhancing microbial metabolite and enzyme production: current strategies and challenges. Front Microbiol. 2014;5:718.

17. Shen B. Polyketide biosynthesis beyond the type I, II and III polyketide synthase paradigms. Curr Opin Chem Biol. 2003;7:285-95.

18. Bentley R. From miso, saké and shoyu to cosmetics: a century of science for kojic acid. Nat Prod Rep. 2006;23:1046-62.

19. Terabayashi Y, Sano M, Yamane N, Marui J, Tamano K, Sagara J, Dohmoto $\mathrm{M}$, Oda K, et al. Identification and characterization of genes responsible for biosynthesis of kojic acid, an industrially important compound from Aspergillus oryzae. Fungal Genet Biol. 2010:47:953-61.

20. Marui J, Yamane N, Ohashi-Kunihiro S, Ando T, Terabayashi Y, Sano M, Ohashi S, Ohshima E, et al. Kojic acid biosynthesis in Aspergillus oryzae is regulated by a $\mathrm{Zn}(\mathrm{II})(2) \mathrm{Cys}(6)$ transcriptional activator and induced by kojic acid at the transcriptional level. J Biosci Bioeng. 2011;112:40-3.

21. Takaya T, Koda R, Adachi D, Nakashima K, Wada J, Bogaki T, Ogino C, Kondo A. Highly efficient biodiesel production by a whole-cell biocatalyst employing a system with high lipase expression in Aspergillus oryzae. Appl Microbiol Biotechnol. 2011;90:1171-7.

22. Watanabe A, Fujii I, Sankawa U, Mayorga ME, Timberlake WE, Ebizuka Y. Reidentification of Aspergillus nidulans wA gene to code for a polyketide synthase of naphthopyrone. Tetrahedron Lett. 1999:91-4.

23. Mizutani O, Kudo Y, Saito A, Matsuura T, Inoue H, Abe K, Gomi K. A defect of LigD (human Lig4 homolog) for nonhomologous end joining significantly improves efficiency of gene-targeting in Aspergillus oryzae. Fungal Genet Biol. 2008;45:878-89.

24. Yamada O, Lee BR, Gomi K. Transformation system for Aspergillus oryzae with double auxotrophic mutations, niaD and SC. Biosci Biotechnol Biochem. 1997:61:1367-9.

25. Tamano K, Satoh Y, Ishii T, Terabayashi Y, Ohtaki S, Sano M, Takahashi T, Koyama Y, et al. The beta-1,3-exoglucanase gene exgA (exg1) of Aspergillus oryzae is required to catabolize extracellular glucan, and is induced in growth on a solid surface. Biosci Biotechnol Biochem. 2007;71:926-34.

26. Amberg DC, Botstein D, Beasley EM. Precise gene disruption in Saccharomyces cerevisiae by double fusion polymerase chain reaction. Yeast. 1995;11:1275-80

27. Tamano K, Sano M, Yamane N, Terabayashi Y, Toda T, Sunagawa M, Koike H, Hatamoto $\mathrm{O}$, et al. Transcriptional regulation of genes on the non-syntenic blocks of Aspergillus oryzae and its functional relationship to solid-state cultivation. Fungal Genet Biol. 2008;45:139-51.

\section{Publisher's Note}

Springer Nature remains neutral with regard to jurisdictional claims in published maps and institutional affiliations.

Ready to submit your research? Choose BMC and benefit from:

- fast, convenient online submission

- thorough peer review by experienced researchers in your field

- rapid publication on acceptance

- support for research data, including large and complex data types

- gold Open Access which fosters wider collaboration and increased citations

- maximum visibility for your research: over $100 \mathrm{M}$ website views per year

At $\mathrm{BMC}$, research is always in progress.

Learn more biomedcentral.com/submissions 